\title{
Behind the Masks: Experiences of Mental Health Practitioners of Color During the COVID-19 Pandemic
}

\author{
Adriana S. Miu ${ }^{1}$ (D) - Jessica R. Moore ${ }^{1}$ \\ Received: 24 November 2020 / Accepted: 18 February 2021 / Published online: 3 March 2021 \\ (C) Academic Psychiatry 2021
}

Systemic racism is deeply rooted in America. The COVID-19 pandemic further brought to light the racial disparities for Black, Indigenous, and People of Color (BIPOC), ranging from higher rates of COVID-19 infections and mortality to racism $[1,2]$. BIPOC communities face systemic inequalities due to lack of healthcare access to well-resourced hospitals, testing availabilities, and staff with linguistic and cultural competencies [3, 4]. BIPOC individuals also likely have a greater exposure to COVID-19 due to financial dependence on essential work and living in close quarters where social distancing is not possible $[2,5]$. Meanwhile, anti-Asian hate crimes, murders of unarmed Black people, and racial profiling have heightened during this pandemic [2].

Although there are reports of the disproportionate impact of COVID-19 on BIPOC communities [6], not much is known about the frontline experiences of BIPOC mental health professionals who heal people's traumatic experiences from COVID-19 and racism. A diverse mental health workforce can reduce distrust and barriers to mental healthcare for communities of color $[7,8]$, which is crucial given racial health disparities. Here, we share personal experiences as an Asian American psychologist and a Black psychiatrist, to inform the unique challenges for BIPOC professionals during this unprecedented time. We suggest that BIPOC professionals are vulnerable to racism and racial trauma, increased burnout and vicarious trauma, and systemic burden. To meet their needs and thereby support BIPOC communities, we recommend changes at the systemic, institutional, and individual levels to reach the roots of systemic racism.

Adriana S. Miu

adriana.miu@utsouthwestern.edu

1 University of Texas Southwestern Medical Center, Dallas, TX, USA

\section{Personal Experiences}

\section{Perspective of an Asian American Psychologist}

During this pandemic, my profession and achievements could not shield me from racism. I am a 1.5-generation immigrant, and I have lived here most of my life and worked as a contributing member of the society. And yet, I am a perpetual foreigner who has been told to "go back to where I came from."

When politicians labeled COVID-19 as the "Chinese virus," they blamed Asian Americans for spreading the virus [2]. Over 1500 anti-Asian hate crimes, and xenophobia were reported within 1 month of the onset of the pandemic in the USA [9]. I watched news videos of Asian Americans being attacked indiscriminately in the public, and my family and I looked like them! I was afraid of going to places alone. I also worried about the safety of my friends and family. This fear was exacerbated by public health messages that only sick people should wear masks. I felt torn about wearing masks early in the pandemic. I knew from the SARS epidemic that masks could protect me from COVID-19 but could also harm me if others saw me as a "virus spreader" who was sick. The Chinese proverb "silence is gold" has been my survival strategy for covert racism. I decided to shrug off racism, focus on work, and not make waves. However, in the face of overt attacks, there is constant fear.

I sat with these worries inside me and tried to maintain composure as a psychotherapist. With my few Asian patients, I shared their helplessness and anxiety about xenophobia. All I could offer was my presence and validation. I supported them in finding that small ounce of control to increase their sense of safety. With other patients of color, we processed their experiences of racism, and I focused on listening and validating their distress. Their race-based stress should be seen as an external reality, not as evidence of a psychiatric diagnosis. Day after day, this all became very heavy. As a result, I have been reminded of systemic racism in therapy sessions while I carried anxiety about xenophobia for my loved ones and me. 
However, I kept on going. Because Asians are less likely to seek mental healthcare due to stigma and language barrier [10], I conducted additional seminars and presentations in hope that I could reach those who do not seek help. It is hard to decline a request when there is taboo in mental health and a shortage of Asian American therapists.

\section{Perspective of a Black Psychiatrist}

I identify as a Black woman and an early career psychiatrist. Since my first day in medical school, these identities have often seemed in constant conflict. Like many Black psychiatrists before me [11], I have grappled with imposter syndrome - a persistent self-doubt of being a fraud whose achievements are due to luck or erroneous evaluations [12]. I felt that I did not belong in the predominately White spaces of Medicine. During the pandemic, the higher rates of COVID-19 deaths and the growing list of unarmed Black men and women who have died at the hands of police violence have only heightened these feelings. There was an implicit message in society that Black lives did not matter.

Following the death of George Floyd, I intentionally avoided the news as I tried to power my way through the next few days of work until I could find time to process another death. Meanwhile, I was carrying a full load of patients, and I knew it was particularly important for me to be there for my Black patients and the community. They needed me. In between patients, I waited for carefully crafted emails from my institution about the need for unity and support for diversity and inclusion. I did not think a well-intentioned email could truly address the hurt I was feeling or the systematic interventions I so desired. The following week, I promptly ended a session with a patient in order to attend a virtual kneel-in to honor George Floyd's death. I cried through the call, wiped my tears, and then quickly ended the call so I could see my next patient on time. During a virtual clinic meeting soon after, I shakily typed in a skype chat box that I was feeling isolated. The support was immediate. However, I instinctively worried about being unprofessional. I made a final plea to my colleagues to not take this as an act of unprofessionalism, and I returned to seeing patients a few minutes later.

In the following months, I have been both tasked with and volunteered for the work of addressing race and racism. I am working on our department's newly created AntiRacism Task Force, giving presentations about Black mental health, and supporting faculty development training on the mistreatment of trainees. Throughout the pandemic, I have been more deliberate than usual to navigate through the long-held destructive images of Black women described by West [13]. I have been careful to smile enthusiastically so that no one will perceive me as angry or divisive. I have offered quick reassurances to colleagues that I am doing well to avoid being seen as overly emotional. I have said yes to more projects than necessary to remind myself and others that I belong in the midst of isolation. In truth, I am working, but I am tired. It is hard to rest when I know I am so needed in the Black community and in institutional diversity efforts.

\section{Major Challenges}

These personal experiences highlight the distinct challenges faced by mental health professionals of color during the pandemic: (1) vulnerability to racism and racial trauma, (2) increased vicarious trauma and burnout, and (3) disproportionate burden and responsibilities.

\section{Vulnerability to Racism and Racial Trauma}

Race is one of the most salient attributes. Behind the masks worn during COVID-19, BIPOC mental health professionals are still identifiable as people of color. Regardless of their profession, some BIPOC professionals have experienced racism and threats, which indicates that they are just as vulnerable to racism as the broader communities of color $[14,15]$. Additionally, the concern goes beyond personal safety. BIPOC professionals may also worry about their family, friends, and communities being the next victims of racial hate crimes or COVID-19 infection. In their work, BIPOC mental health professionals must also navigate the potential for racism during a patient interaction while they strive to remain professional and therapeutic for patients from a variety of backgrounds [16].

Although BIPOC professionals are likely to be well-versed in self-care and coping, they can still be vulnerable to racial trauma. Racial trauma refers to real or perceived threats of harm and injury, humiliation, or witnessing harm to others in the community [17]. Professionals of color are exposed to all forms of race-based stress, ranging from direct racism in society, patients' discrimination, or vicarious exposure from news and patients' accounts. [18] As they witness higher rates of COVID-19 infections and racism in their communities, it can reinforce the implicit message that people of color, including BIPOC professionals themselves, are inferior in society and that their lives do not matter as much. It is difficult to heal from the wounds of racial trauma if society continues to oppress against BIPOC communities. It is as if a wound continues to be stabbed again while trying to heal. Therefore, minority mental health professionals can only cope with racial trauma temporarily, but the full healing depends on when systemic oppression ends.

\section{Increased Vicarious Trauma and Burnout}

BIPOC professionals may experience greater burnout from compassion fatigue and vicarious trauma, which refers to 
emotional distress from continuous exposure to victims of trauma in their occupations [19]. BIPOC providers are further exposed to vicarious trauma when they work closely to support patients of color. Providers listen and learn about intimate details of patients' racism, difficulties with social distancing inside close quarters, or dilemmas about sending their children to school as they continue high-risk essential work. Their work requires them to be emotionally available to all kinds of negativity and suffering $[19,20]$. This increases vicarious trauma beyond the original racial disparities in society.

Further, there may be an increased sense of responsibility to rise above the occasion and be much more available to support communities of color. Because COVID-19 infections and deaths are more prevalent in BIPOC communities, rates of depression, grief, and distress also rise among these groups [2]. Research has shown strong distrust and stigma in mental healthcare in BIPOC communities, and therapist matching based on race can mitigate these barriers [7, 8]. BIPOC professionals can bring in the expertise of cultural understanding and language that benefit patients of color. However, BIPOC providers have long been underrepresented in the mental health profession $[21,22]$, despite the need for their cultural representations and skills during the pandemic. This likely intensifies burnout for BIPOC professionals who are already "stretched thin" when they cannot share the responsibilities with other mental health professionals.

\section{Disproportionate Burden by Minority Tax}

In addition to bearing more burden in caring for BIPOC communities, BIPOC professionals may also experience additional responsibilities in addressing racism in their workplace. Because there is systemic underrepresentation of minorities in the mental health workforce, BIPOC providers may be asked to be diversity experts or cultural conduits. This constitutes a "minority tax," being called to take on extra responsibilities in the name of diversity [23]. These responsibilities may include participation or leadership in a racial justice task force, providing mentorship, or delivering didactic lectures. Although these opportunities are meaningful, minority professionals are already "stretched thin" with high levels of burnout. It can be difficult to say no when they are passionate about antiracism or reducing minority communities' barriers to healthcare, or when the requests come from their employers. These opportunities, where racial health disparities or racism may be discussed, could further retraumatize BIPOC professionals. At a time when self-care is paramount, the minority tax can be detrimental [24].

Unfortunately, BIPOC professionals often lack adequate mental health support and role models to help navigate these race-based burdens. With only about $2-5 \%$ of BIPOC mental health providers in the workforce $[21,22]$, it is likely challenging for BIPOC mental health professionals to obtain care or seek advice from a provider or mentor of color who would understand the nuances of their struggles.

Without dedicated support, BIPOC professionals may not ask for help, especially when they struggle with imposter syndrome $[11,12]$. Afraid of appearing inadequate or incompetent, it may be difficult to acknowledge their struggles and request help. This imposter syndrome can be exacerbated during the pandemic when BIPOC communities have been pitted as "less than equal" in COVID-19 care and in racial oppressions. When BIPOC lives do not appear to matter in a larger society, this implicit message can deepen underlying selfdoubt that existed prior to the pandemic. Imposter syndrome may further worsen among BIPOC groups who wonder whether they are hired as a token rather than on merit. Given this context, BIPOC professionals may not feel comfortable voicing their struggles to other professionals who cannot relate to their experiences.

Outside of work, they may not be comfortable voicing their own struggles in their social network among friends, family, and community. Because their own community may also experience racial injustice and COVID-19-related stress, minority professionals may feel more pressured to appear collected and serve as the anchoring rock for their community during the pandemic.

\section{Recommendations}

To combat racism, burnout, and disproportionate burden on BIPOC professionals during the pandemic, we need policies and actions from all levels (individual, institution, and system). It is insufficient and unsustainable to rely on changes at just one level. Below are evidence-based recommendations informed by the racial trauma and burnout literature $[19,20$, $25,26]$.

\section{Individual}

On an individual level, it is important to frequently check in with the self. BIPOC professionals dedicate themselves to a helping profession, but they cannot optimally help patients unless they feel balanced and present. In response to racial trauma, reactions can range from intense sadness and fear to blunted affect, numbness, and avoidance [27]. Even if one appears unaffected, there may be underlying confusion and numbness. Therefore, it is important to practice selfacceptance and compassion, to recognize that one may be particularly burdened, and it is okay to not be okay. BIPOC providers need to recognize that they are humans and also need social support and understanding from others. It is important that they give themselves the permission to take breaks and take off the "professional hat" when not working. Selfcare activities may include exercise, hobbies, regular breaks, 
social support, and peer consultations with like-minded values about social justice.

Racial health disparities and systemic racism do not have an immediate solution. However, one can address burnout by practicing self-awareness and gratitude. BIPOC providers may set a daily intention exercise by asking, "Why am I doing what I am doing?" or "What is my one intention today?" [19, 20]. By setting a specific goal of the day (e.g., be present or appreciative), one may become centered by personal values despite external chaos. Additionally, BIPOC providers may also practice gratitude daily. When a BIPOC patient shares their experience of racism, professionals can appreciate the trust that their patients develop in them. It is a testament to the importance of BIPOC professionals' roles in reducing stigma and barriers to mental healthcare in communities of color. Providers can also identify three things they are grateful for or one thing that they have done well daily. These daily practices will help reinforce the idea that BIPOC providers' work matters, even if they cannot immediately solve the systemic disparities.

Additionally, the "minority tax" associated with this work can be damaging. BIPOC providers should self-reflect and choose social justice projects that align with their own personal values, professional goals, and interests. By declining projects that do not align with these values, BIPOC professionals may find more enjoyment and a sense of accomplishment from the chosen projects. If professionals of color acknowledge the limitations of their abilities to fix the injustice and cope with race-based stress, they can better sustain their professional work serving communities of color.

\section{Institution}

Institutions first need to create a safe space for BIPOC professionals within the organization. For example, leadership should make public statements denouncing racial disparities and commit to ongoing anti-racist efforts to examine and reflect on practices. Institutions should consider a selfassessment on recruitment and retention of BIPOC professionals, systemic barriers for patients of color seeking care, departmental protocols on addressing racism in both patient and collegial interactions, available supports for BIPOC providers, and anti-racist education initiatives. Institutions should consider their role in the barriers that may contribute to COVID-19 health disparities, such as access to care, treatment of underlying health conditions, and protocols for access to testing for BIPOC communities. Institutions may consider consulting with external diversity and inclusion specialists who can provide an objective evaluation and recommendations. This would also help reduce minority tax placed on BIPOC providers. When BIPOC professionals are doing institutional work on diversity, institutional leadership should ensure that there is adequate protected time for this work and their efforts are appropriately recognized.

Institutions should take an active role in supporting BIPOC professionals given the likelihood of imposter syndrome and barriers to help-seeking. To counter the oppressive rhetoric of BIPOC individuals as "inferior," institution leaders can acknowledge and recognize BIPOC providers as valued members of the institution. Institutions may also increase flexibility for the BIPOC providers by allowing additional time for selfcare or more control over their workload and schedule. BIPOC professionals may not seek emotional support from employee assistance programs or fellow colleagues where they could be judged for needing help. It would be helpful to provide external resources, such as confidential mental health support or mentors (preferably BIPOC) outside of the institutions.

\section{Structural System}

Structural changes are needed in professional and academic societies representing psychiatrists, psychologists, social workers, or other mental health professionals. First, in their mission statements, these systems should condemn racism and reduce systemic inequality in both clinical care and organizations. Second, the burden of supporting BIPOC communities' mental health should not only fall on the shoulders of BIPOC providers, even if they may have more credibility or expertise. Professional societies and training programs should require dedicated coursework or continuing education on diversity and systemic barriers to mental healthcare for communities of color. To sustain multiculturalism education, professional organizations should commit to ongoing training that does not end when a social movement wanes. Training should also focus on clinical practice beyond coursework, so that all providers are challenged to work with patients of color and gain more cultural humility. It is important to emphasize that professionals of every racial and ethnic group, including those who are White, are responsible for addressing racial disparities in mental health.

Lastly, it is not sufficient to hire diverse staff and providers, although it is a beginning step. BIPOC providers need a voice, as well as a face, lest they be merely a token of representation. Diversity should be respected and celebrated as a path to excellence, rather than a problem to fix [28]. Myers [29] once stated, "Diversity is being invited to the party. Inclusion is being asked to dance." Even after achieving diverse representation, our systems need to expand on inclusion. Blackmon [30] further suggested, "Belonging is being able to dance how you want - and equity is having a turn picking the DJ." Not only should BIPOC individuals be invited to apply for leadership or professional opportunities, but also be given the opportunity to thrive once in these positions. Their experiences should be viewed as valuable and valid, and they should 
have a voice in policy and decision-making. It is important to create a culture that fosters a sense of belonging and reduces tokenism. Professional organizations may address issues of diversity in the pipeline to the mental health workforce by exposing BIPOC middle and high school students to careers in mental health. They may challenge imposter syndrome in BIPOC college students applying to medical or graduate school and provide wellness resources to trainees.

\section{Conclusion}

BIPOC mental health professionals experience additional challenges during the COVID-19 pandemic due to racial health disparities and increased racism. Their profession does not make them immune to racial injustice. Instead, they likely experience disproportionately more burnout and responsibilities. Given these challenges, it is important to address these issues at all levels - individual, institution, and system. Systemic racial disparities have always been there, but only heightened during the COVID-19 pandemic. Everyone should be responsible for these systemic issues, not just the BIPOC professionals. Therefore, we should not only fight against systemic racism during the COVID-19 pandemic but rather continue this advocacy for as long as racial disparities last.

Acknowledgements We are grateful to Adam Brenner and Lia Thomas for their feedback and suggestions on this commentary.

\section{Declarations}

Disclosures On behalf of all authors, the corresponding author states that there is no conflict of interest.

\section{References}

1. Center for Disease Control and Prevention Coronavirus disease 2019 (COVID-19) in the US [Internet]. CDC; 2020 [updated 2020 ; cited 2020 Nov 18]. Available from: https://covid.cdc.gov/ covid-data-tracker/.

2. Liu SR, Modir S. The outbreak that was always here: racial trauma in the context of COVID-19 and implications for mental health providers. Psychol Trauma Theory Res Pract Policy. 2020;12(5):439-42.

3. Williams DR, Lawrence JA, Davis BA. Racism and health: evidence and needed research. Annu Rev Public Health. 2019;40: 105-25.

4. Tai DB, Shah A, Doubeni CA, Sia IG, Wieland ML. The disproportionate impact of COVID-19 on racial and ethnic minorities in the United States. Clin Infect Dis. 2020 Jun;20.

5. Bureau of Labor Statistics. The employment situation October 2020 [Internet]. Washington DC: Bureau of Labor Statistics; 2020 [updated 2020; cited 2020 Nov 18]. Available from: https://www.bls. gov/news.release/empsit.t02.htm
6. Stokes EK, Zambrano LD, Anderson KN, Marder EP, Raz KM, Felix SE, et al. Coronavirus disease 2019 case surveillanceUnited States, January 22-May 30, 2020. MMWR Morb Mortal Wkly Rep. 2020;69:759-65 Available from: https://www.cdc.gov/ mmwr/volumes/69/wr/mm6924e2.htm.

7. McGuire TG, Miranda J. New evidence regarding racial and ethnic disparities in mental health: policy implications. Health Aff. 2008;27(2):393-403.

8. Meyer OL, Zane N. The influence of race and ethnicity in clients' experiences of mental health treatment. J Community Psychol. 2013;41(7):884-901.

9. Choi C, Kulkarni MP. In one month, STOP AAPI HATE receives almost 1500 incident reports of verbal harassment, shunning, and physical assaults. Los Angeles: Asian Pacific Policy \& Planning Council; 2020. [updated 2020 Apr 23; cited 2020 Nov 18]. A va i $1 \mathrm{able}$ f r o m: ht t p : / / w w w. asianpacificpolicyandplanningcouncil.org/wp-content/uploads/ Press_Release_4_23_20.pdf

10. Huang CY, Zane N. Cultural influences in mental health treatment. Curr Opin Psychol. 2016;8:131-6.

11. Thomas LA. A dark spot on a white canvas: thoughts on being a black academic psychiatrist. Acad Psychiatry. 2019;43(4):455-6.

12. Clance PR, Imes SA. The imposter phenomenon in high achieving women: dynamics and therapeutic intervention. Psychother: Theory, Res Pract. 1978;15(3):241-7.

13. West CM. Mammy, Sapphire, and Jezebel: historical images of Black women and their implications for psychotherapy. Psychother Theory Res Pract Train. 1995;32(3):458-66.

14. Cheng TL, Conca-Cheng AM. The pandemics of racism and COVID-19: danger and opportunity. Pediatr. 2020;146 (5).

15. Peterson NB, Friedman RH, Ash AS, Franco S, Carr PL. Faculty self-reported experience with racial and ethnic discrimination in academic medicine. J Gen Intern Med. 2004;19(3):259-65.

16. Goldenberg MN, Cyrus KD, Wilkins KM. ERASE: a new framework for faculty to manage patient mistreatment of trainees. Acad Psychiatry. 2019;43(4):396-9.

17. Carter RT. Racism and psychological and emotional injury: recognizing and assessing race-based traumatic stress. Couns Psychol. 2007;35(1):13-05.

18. Nunez-Smith M, Curry LA, Bigby J, Berg D, Krumholz HM, Bradley EH. Impact of race on the professional lives of physicians of African descent. Ann Intern Med. 2007;146(1):45-51.

19. Greenberg TM. Vicarious trauma and self-care for the trauma therapist. In Greenberg TM, editor. Treating complex trauma [Internet]. $1^{\text {st }}$ ed. New York City: Springer, Cham; 2020.p. [191-211]. Available from: https://doi.org/10.1007/978-3-03045285-8_10, 2020

20. Lipsky L, Burk C. Trauma stewardship: an everyday guide to caring for self while caring for others. San Francisco, CA: BerrettKoehler Publishers; 2009.

21. American Psychological Association. Demographics of U.S. psychological workforce [Internet]. Washington (DC): American Psychological Association; 2017 [updated 2020; cited 2020 Nov 10]. Available from: https://www.apa.org/workforce/datatools/demographics.aspx.

22. Association of American Medical Colleges. Table 13, Practice specialty, males by race/ethnicity. Washington: Association of American Medical Colleges; 2018. [cited 2020 Nov 10]. Available from: https://www.aamc.org/data-reports/workforce/ data/table-13-practice-specialty-males-race/ethnicity-2018

23. Rodriguez JE, Campbell KM, Pololi LH. Addressing disparities in academic medicine: what is minority tax? BMC Med Educ. 2015; 15:6.

24. Zambrana RE, Valdez RB, Pittman CT, Bartko T, Weber L, ParraMedina D. Workplace stress and discrimination effects on the 
physical and depressive symptoms of underrepresented minority faculty. Stress Health. 2020.

25. Morse G, Salyers MP, Rollins AL, Monroe-DeVita M, Pfahler C. Burnout in mental health services: a review of the problem and its remediation. Adm Policy Ment Health Ment Health Serv Res. 2012;39(5):341-52.

26. Bryant-Davis T. Healing requires recognition: the case for racebased traumatic stress. Couns Psychol. 2007;35(1):135-43.

27. Center for Substance Abuse Treatment. Trauma-Informed Care in Behavioral Health Services. Chapter 3, Understanding the impact of trauma. Rockville: Substance Abuse and Mental Health Services Administration (US); 2014. Available from: https://www.ncbi.nlm. nih.gov/books/NBK207191/
28. Nivet MA. Commentary: Diversity 3.0: a necessary systems upgrade. Acad Med. 2011;86(12):1487-9.

29. Myers V. Diversity is being invited to the party. Inclusion is being asked to dance [Internet]. The Verna Myers Company; 2020 [cited 2020 Nov 10]. Available from: https://www.vernamyers.com/

30. Blackmon D. Who we are [Internet]. Inclusion Design Group; 2020 [cited 2020 Nov 10] Available from: https://inclusiondesign.com/ who-we-are/

Publisher's Note Springer Nature remains neutral with regard to jurisdictional claims in published maps and institutional affiliations. 